\title{
Results of effectiveness trials of PRO 2000 gel: lessons for future microbicide trials
}

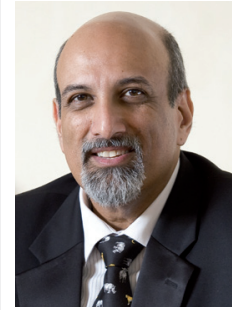

“Despite numerous scientific, ethical and methodological challenges, microbicides provide real potential to influence the course of the HIV epidemic as they could fill an important gap for women-initiated prevention methods."

\section{Salim S Abdool Karim}

Centre for the AIDS Program of Research in South Africa (CAPRISA), University of KwaZulu-Natal, Private Bag X7, Congella 4013, South Africa = Tel.: +27 312604550 = Fax: +27 312604549 = karims1@ukzn.ac.za

In sub-Saharan Africa there is a disproportionate burden of HIV in young women [1]. Microbicides are products that can be applied to the vagina or rectum with the intention of reducing the acquisition of sexually transmitted infections, including HIV, in women. If proven to be effective at reducing incident HIV infections, microbicides could fill an important HIV prevention gap, especially for those women who are unable to successfully negotiate mutual monogamy or condom use. Microbicides have the potential to alter the trajectory of the HIV epidemic in southern Africa [2].

To date, every effectiveness trial of candidate microbicides has shown no impact on HIV acquisition [3-8]. Indeed, trials of two products, COL-1492 [7] and cellulose sulfate [8], showed trends toward increased risk of HIV infection. The recently completed HIV Prevention Trials Network (HPTN) 035 trial of $0.5 \%$ PRO 2000 gel demonstrated a modest $30 \%$ reduction in HIV incidence in a four-arm study of 3099 women with $87 \mathrm{HIV}$ infections in the PRO 2000 and placebo arms of this trial (risk ratio: 0.70) [9]. However, this effect on HIV infection was not statistically significant $(\mathrm{p}=0.1)$. Subsequent findings from the almost threefold larger Microbicide Development Program (MDP) 301 trial, which had 0.5\% PRO 2000 and placebo groups comprising 6268 women with $253 \mathrm{HIV}$ infections, showed that $0.5 \%$ PRO 2000 had no protective effect against HIV infection (risk ratio: 1.05) [101].

\section{How should the different outcomes from these two trials be interpreted?}

There are three scenarios worthy of exploration. In scenario one, where PRO 2000 has no protective effect against HIV infection, both the MDP 301 trial and HPTN 035 trial results could be interpreted as being consistent with this outcome since the $95 \%$ confidence intervals of the risk ratios for both trials included zero effect. In this case, the point estimate of 5\% increase in HIV incidence observed in the $0.5 \%$ PRO 2000 group of the MDP 301 trial and the point estimate of $30 \%$ reduction in HIV incidence observed in the $0.5 \%$ PRO 2000 group of the HPTN 035 trial is variability due purely to chance. While the observed effect in the HPTN 035 trial is sufficiently distant from zero effect to suggest that PRO 2000 may have some benefit, the p-value of 0.10 meant that there was still an unacceptably high likelihood that PRO 2000 is not effective at all.

In scenario two, PRO 2000 has a moderate protective effect against HIV infection. A moderate effect of up to $18 \%$ protection is not inconsistent with the results of the two trials as this level of protection is within the upper bounds of the 95\% confidence intervals of the risk ratios from both studies.

In scenario three, PRO 2000 has a substantial protective effect against HIV infection of, say, $55 \%$. In this scenario, both trials may have produced underestimates of the 'true' effect. The upper bounds of the $95 \%$ confidence intervals of the risk ratios from both trials exclude this level of effect. While this scenario is unlikely, if it did occur, the underestimate would be most likely due to suboptimal adherence. Inadequate adherence, especially in relation to exposure to HIV, is one of the most serious threats that could undermine a microbicide trial's ability to demonstrate an accurate level of efficacy of the product [10]. In microbicide trials, the effectiveness of a product is dependent on both the efficacy of the product and the participants' willingness and ability to use the product as instructed. For example, trials with only $50 \%$ adherence will be able to demonstrate,

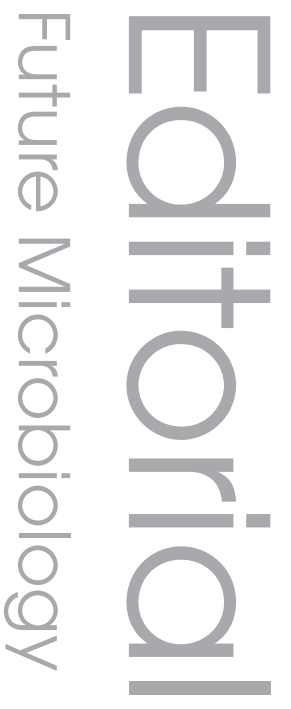

\footnotetext{
${ }^{66}$ Microbicides have the potential to alter the trajectory of the HIV epidemic in southern Africa."
}

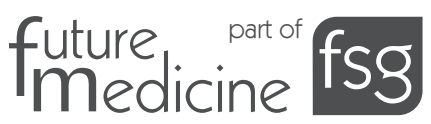




\begin{tabular}{c}
\hline "...the results of the \\
HPTN 035 and MDP 301 \\
trials suggest that, \\
although safe, 0.5\% PRO \\
2000 gel may have little or \\
no effect on reducing a \\
woman's risk of \\
HIV infection..."
\end{tabular}

\section{Bibliography}

1. Abdool Karim Q: Heterosexual transmission of HIV - the importance of a gendered perspective in HIV prevention. In: HIVIAIDS in South Africa. Abdool Karim SS, at most, half the true efficacy of a short-acting product such as PRO 2000. Measuring adherence is also challenging and although current clinical trials all include some form of assessment of product use, measures such as self-reported behavior can sometimes be unreliable.

Each of these scenarios provides a perspective on how the results of the HPTN 035 and MDP 301 trials can be interpreted. Taken together, the results of the HPTN 035 and MDP 301 trials suggest that, although safe, 0.5\% PRO 2000 gel may have little or no effect on reducing a woman's risk of HIV infection during typical use and that this product is not worth pursuing further as a potential microbicide. This outcome was particularly disappointing as $0.5 \% \mathrm{PRO}$ 2000 has potent activity against HIV in vitro [11-13] and in animal models for vaginal HIV transmission [14,15].

\section{What lessons can be learnt for future microbicides trials?}

First, these results raise a question about the usefulness of animal models that showed PRO 2000 had a protective effect. Second, these results highlight the fundamental challenges faced in determining which products warrant proceeding into human efficacy trials in the absence of a surrogate marker of protection. Third, adherence and measurement thereof is fundamentally important in microbicide trials in order to exclude scenario three. Studies assessing new formulations, such as vaginal rings, to improve adherence are needed. Fourth, both trials achieved their objective; the HPTN 035 study as a screening Phase IIb trial and the MDP 301 study as a Phase III trial have together addressed the question of whether PRO 2000 is effective in preventing HIV infection following typical use. Finally, as much as we need a series of human studies looking at effectiveness of products, we also need to build into these studies a program of basic science research to identify potential surrogate markers of protection. Without a validated criterion to decide whether a product should proceed, the microbicides field is hamstrung with doing either relatively large expensive screening trials or proceeding straight to even larger and often substantially more expensive Phase III trials.
Despite numerous scientific, ethical and methodological challenges, microbicides provide real potential to influence the course of the HIV epidemic as they could fill an important gap for women-initiated prevention methods. The joint results of the HPTN 035 and MDP 301 trials have closed a chapter in microbicide development by being the last studies on polymers as candidate microbicides. Attention is now firmly focused on antiretroviral agents for topical use as candidate microbicides with results on tenofovir gel due for release in mid 2010.

Questions that remain to be answered include:

- When an effective microbicide is found, what data will be needed to address the main obstacles to widespread use; for example, safety in pregnancy, long-term safety, safety in very high-frequency use, approaches to increase over-the-counter access to the product, messaging to promote uptake and disposable, low-cost applicators?

- What are the most appropriate correlates of protection?

- Which formulation of microbicides will be most acceptable (e.g., gel, vaginal ring or film)?

- How should a microbicide product be formulated to promote adherence?

The interpretation of these upcoming results on tenofovir gel will benefit enormously from the lessons learnt from the HPTN 035 and MDP 301 trials.

\section{Financial \& competing interests disclosure}

SS Abdool Karim is Protocol Chair of the HPTN 035 trial which was supported by the NIH (grant \# U01AI46749 and U01A1068633) and Co-Principal Investigator of the CAPRISA 004 trial of tenofovir gel, which was supported by United States Agency for International Development (\# GPO-A-00-05-0002200, contract \# 132119) and LIFElab, a South African government biotechnology agency. The author has no other relevant affiliations or financial involvement with any organization or entity with a financial interest in or financial conflict with the subject matter or materials discussed in the manuscript apart from those disclosed.

No writing assistance was utilized in the production of this manuscript.

microbicide's sexually transmitted infection efficacy may contribute to the HIV effectiveness measured in

Phase 3 microbicide trials. J. Acquir. Immune Defic. Syndr. 48(4), 460-467 (2008). 
3. Feldblum PJ, Adeiga A, Bakare R et al:: SAVVY vaginal gel (C31G) for prevention of HIV infection: a randomized controlled trial in Nigeria. PLoS ONE 3(1), e1474 (2008).

4. Kreiss J, Ngugi E, Holmes K et al: : Efficacy of nonoxynol 9 contraceptive sponge use in preventing heterosexual acquisition of HIV in Nairobi prostitutes. JAMA 268(4), 477-482 (1992).

5. Roddy RE, Zekeng L, Ryan KA, Tamoufe U, Weir SS, Wong EL: A controlled trial of nonoxynol 9 film to reduce male-to-female transmission of sexually transmitted diseases. N. Engl. J. Med. 339(8), 504-510 (1998).

6. Skoler-Karpoff S, Ramjee G, Ahmed K et al.: Efficacy of Carraguard for prevention of HIV infection in women in South Africa: a randomised, double-blind, placebo-controlled trial. Lancet 372(9654), 1977-1987 (2008).

7. Van Damme L, Ramjee G, Alary M et al.: Effectiveness of COL-1492, a nonoxynol-9 vaginal gel, on HIV-1 transmission in female sex workers: a randomised controlled trial. Lancet 360, 971-977 (2002).

8. Van Damme L, Govinden R, Mirembe FM et al: : Lack of effectiveness of cellulose sulfate gel for the prevention of vaginal HIV transmission. N. Engl. J. Med. 359(5), 463-472 (2008).

9. Abdool Karim SS, Coletti A, Richardson B et al.: Safety and effectiveness of vaginal microbicides BufferGel and PRO $2000 \mathrm{Gel}$ for the prevention of HIV infection in women: results of the HPTN 035 trial. Presented at: Conference on Opportunistic Infections and Retroviruses. Montreal, Canada, 8-11 February 2009 (Abstract 48lb).

10. Methodological Challenges in Biomedical HIV Prevention Trials. Lagakos SW, Gable AR (Eds). National Academy of Sciences, Washington DC, USA (2008).

11. Dezzutti CS, James VN, Ramos A et al.: In vitro comparison of topical microbicides for prevention of human immunodeficiency virus type 1 transmission. Antimicrob. Agents Chemother. 48(10), 3834-3844 (2004).

12. Greenhead P, Hayes P, Watts PS, Laing KG, Griffin GE, Shattock RJ: Parameters of human immunodeficiency virus infection of human cervical tissue and inhibition by vaginal virucides. J. Virol. 74(12), 5577-5586 (2000).

13. Scordi-Bello IA, Mosoian A, He C et al.: Candidate sulfonated and sulfated topical microbicides: comparison of anti-human immunodeficiency virus activities and mechanisms of action. Antimicrob. Agents Chemother. 49(9), 3607-3615 (2005).

14. Lewis MG, Wagner W, Yalley-Ogunro J, Greenhouse J, Profy AT: Efficacy of PRO $2000 \mathrm{Gel}$ in a macaque model for vaginal HIV transmission. Presented at: Microbicides 2002. Antwerp, Belgium, 12-15 May 2002 (Abstract A-191).
15. Weber J, Nunn A, O'Connor T et al.: 'Chemical condoms' for the prevention of HIV infection: evaluation of novel agents against SHIV(89.6PD) in vitro and in vivo. AIDS 15(12), 1563-1568 (2001).

\section{Website}

101. MDP Press release: HIV 'prevention' gel PRO 2000 proven ineffective (2009).

www.mdp.mrc.ac.uk/

\section{Affiliations}

- Salim S Abdool Karim

Centre for the AIDS Program of Research in South Africa (CAPRISA), University of KwaZulu-Natal, Private Bag X7, Congella 4013, South Africa

Tel.: +27312604550

Fax: +27312604549

karims1@ukzn.ac.za

and

Department of Epidemiology, Mailman School of Public Health, Columbia University, 722 West, 168th Street, NY 10032, USA 